\title{
FORECASTING DEMAND FOR A TELEPHONE CALL CENTER
}

\author{
Pavle Bugarčić ${ }^{1}$, Valentina Radojičić ${ }^{2}$ \\ 1,2 University of Belgrade, Faculty of Transport and Traffic Engineering, Vojvode Stepe 305, 11000 \\ Belgrade, Serbia
}

Received 20 April 2020; accepted 11 June 2020

\begin{abstract}
A company can predict satisfactory staffing and scheduling levels, improve service requirements, meet customer satisfaction by forecasting the real number of incoming calls. Accurate forecast of customer demand is crucial for the staffing algorithm of a telephone Call Center. There is a problem of scheduling and re-scheduling the available pool of agents based on updated forecasts, typically made weeks or months in advance. Here, we selected some forecasting methods that can generate both time-varying and stochastic day-to-day demand. This paper proposes three different methods in detail: the Moving Averages method, Simple Exponential Smoothing method, and Additive Holt-Winters method. We presented the forecasting results obtained from an empirical study analyzing real Call Center data by months. Finally, each of these methods was tested and the comparison results are given.
\end{abstract}

Keywords: Call Centers, demand, forecasting, incoming calls, traffic profile.

\section{Introduction}

A Call Center may be the electronic face of the company, and with advancement of technology and trend of digitalization, it has become a vital service for interacting with existing and future customers. In present times, Call Centers have become one of the most important forms of communication between companies and customers. The most common form of Call Centers is inbound Call Centers, where customers initiate calls and Call Center agents respond to those calls. It can be noticed that an increasing number of companies have their own Call Centers, for the best possible provision of services and information to the customers. With the efficient use of Call Centers, customers make significant savings in time, while companies make significantly more efficient operations.

In order to achieve the right balance between quality of service and operational efficiency,
Call Center managers are faced with the problem of determining appropriate staffing levels, weeks or months in advance, based on medium-term forecasts of future incoming demand which is typically both time-varying and stochastic (Gans, Koole and Mandelbaum, 2003). This is the reason why different amounts of human and technical resources of the Call Center are required at different periods. This is mainly related to the optimal number of Call Center agents working simultaneously. The problem arises if the technical and human resources are too small or too large. If the resources are below the required level, then they are not sufficient for quality and timely customer service. On the other hand, if resources are above the required level, there is an underutilization of resources. This problem is noticeable both on a daily, as well as on a weekly, monthly or yearly basis.

Looking at the results of a year measurement period, it could be notes that the intensity

${ }^{1}$ Corresponding author: p.bugarcic@sf.bg.ac.rs 
of the incoming Call Center calls varies throughout the year. Therefore, during periods of increased traffic intensity, it is necessary to provide more technical and human resources, for example, a larger number of Call Center agents to avoid congestion, excessive waiting and/ or rejecting customer calls. On the other hand, during periods of decreased traffic intensity smaller numbers of employed Call Center agents are needed to prevent their underutilization.

First of all, a reliable forecast of incoming calls based on the measurement results used to optimize technical and human resources of a telephone Call Center is needed. Since day-to-day and within the day traffic profiles are very dynamic, the number of incoming calls cannot be modeling by any of the classical mathematical models, such as linear, exponential or logistic method. Better results could be obtained by models which involving seasonal influences, such as the Moving Averages method (Kovačić, 1998). Also, it is possible to apply models that do not include seasonal influences and do not follow any trend, such as the Simple Exponential Smoothing method. Even better results could be obtained by extending the Simple Exponential Smoothing method to include level, trend and seasonal influences. One of the methods that include these extensions is called the Additive Holt-Winters method.

In this paper, we proposed alternative time series methods for forecasting demand in a Call Center. These methods are used to forecast the number of incoming Call Center calls for the next year, based on the measurement results during the last two years. The total number of incoming Call Center calls by month is observed. Several different tests, such as mean absolute percentage error (MAPE), mean absolute error (MAE), and Durbin-Watson (DW) tests are used to compare these methods.

The paper is organized as follows. After the introductory section, section 2 describes types and the most important features of Call Centers. Section 3 describes the applied methods: the Moving Averages method, Simple Exponential Smoothing method, and Additive Holt-Winters method. Our forecasting procedure is described in detail in this section. The proposed methods are demonstrated using a real data set during the last two years, in Section 4. Within this section, the reliability of the forecasts was tested using the MAPE, MAE and DW tests. At the end of this section, the obtained forecasting results are compared, based on these tests. Concluding remarks are given in section 5 .

\section{Call Centers}

A Call Center is a centralized office used for the purpose of receiving a large volume of call requests generated by telephone users. It is preferred and prevalent way for many companies to communicate with their customers. In today's increasingly competitive marketplace, a company's Call Center can become an important source of competitive advantage. It has been recognized that a critical factor in business success in this environment is being able to respond rapidly to customer requests. Indeed, companies with top-performing Call Centers typically have more satisfied customers, lower operating costs, and higher revenue per customer (Radojičić, Marković and Radonjić, 2010).

A Call Center represents several different things: profit center, cost center, major 
source of revenue, major source of customer dissatisfaction, strategic weapon, strategic disadvantage, source of marketing research, etc. The importance of Call Center services varies from company to company, depending on how closely the Call Center works with parent or customer organizations to support the company. For customers, Call Centers are the "voice" of the company.

There are several ways to communicate with customers, depending on type of Call Center. The three major types of Call Centers are: inbound, outbound, and blended. With inbound Call Centers, customers initiate calls. Services provided by inbound Call Centers are customer service, technical support for business or private customers, downloading, entering and processing orders, scheduling appointments, reservations and registration, etc. This type of Call Center is analyzed in this paper. In an outbound Call Center, agents of the company initiate calls to customers. Many companies hire an outside company for the services provided by the outbound Call Center. Some Call Centers can receive calls from customers at the same time and make calls to customers. Such Call Centers are called blended Call Centers.

In addition to this basic division, several other types of Call Centers can be identified. Virtual Call Centers are Call Centers where agents are not located in one location but can be geographically dispersed. The offshore Call Center is an organization that provides customer service in another country. A webenabled Call Center is a Call Center that a customer can call by pressing a button on a website.

Some of the most important features of the Call Center management are:
- Allows incoming phone calls to be properly categorized and qualified without using live agent resources,

- Responds quickly to customer calls;

- Solves a high percentage of customer queries at the very first call, which has good results in terms of customer satisfaction;

- Allows monitoring phone calls, which play an important role in improving and optimizing Call Center efficiency;

- Provides a significant source of revenue to the company;

- Constantly improve business efficiency;

- Allows the company to view the Call Center as a strategic asset, etc.

Accurate forecast of incoming calls is critical for staffing problem optimization. Input data on the number of incoming calls in the previous period is required for the human and technical planning process. The result of the planning process should be corresponding with the optimal number of agents per shift, which is a very complex optimization task. Since the traffic profile of Call Center calls is very dynamic, this complicates the forecasting process. The following section selected some methods that can be very suitable for a reliable forecast of incoming Call Center calls.

\section{Forecasting Methods}

The forecasting process can be divided into the following parts:

- Definition of the problem - the purpose of and assumptions for forecast has to be determined (staffing and scheduling, technical resources, etc);

- Collection of basic data - historical data by day during the whole year. In addition, population and economic growth are studied. The results of recent forecasts are essential facts; 
- Choice of the forecasting method selection of methods are made with regard to the available historical data, time domain, various influencing factors of user behavior (lifestyle, economic situation, occupation, age, personality, etc) and required accuracy;

- Estimation of model parameters - the least squares method can be used to estimate the parameters;

- Evaluation of forecasting results - the obtained results have to be tested; forecast errors are used for the methods comparison;

- Documentation - the forecast has to be presented in an easily understandable format. The result should contain alternative forecasts (optimistic and pessimistic forecast).

The forecasting methods could be divided in the following generalized groups (Brockwell, 2002):

- Time series forecasting methods - it is assumed that development will follow a curve which has been fitted to existing historical data;

- Causal/econometric forecasting methods - it is assumed that explicit relationships between demand and various determining factors are existed - the basic assumption is that these will remain the same in the future;

- Judgmental methods - personal or expert judgment in the forecast; the future will resemble the person's previous knowledge and experience of past developments.

Usually, time series models have been used to model Call Center calls. Its implementation is easy and flexible because it only requires historical observations of necessary variables. Many time series, despite the presence of trends, have seasonality characteristics (Lepojević and Anđelković-Pešić, 2011).

In this paper, we analyzed the measurement results of incoming Call Center calls by months. It could be notice that time series has some seasonal variations but does not follow any distinctive trend. For this reason, the Moving Averages method which includes seasonal influences is our first choice. Then, two methods were chosen from the group of Exponential Smoothing methods: Simple Exponential Smoothing method which does not follow any trend and does not include seasonal influences, and Additive HoltWinters method which supplements the Simple Exponential Smoothing method by introducing trend and seasonal influences.

\subsection{Moving Averages Method}

The Moving Averages method is the most common method used in practice to smooth out short-term fluctuations. A moving average can be thought of as a rolling average in that the average of the last several values of the time series is used to forecast the next value. Moving averages are calculated as the average values of subsets (of $k$ elements) of the input data set (of $n$ elements), with the condition that $k<n$, and represents simple or weighted averages of subsets of the underlying data set (Radojičić and Bakmaz, 2010). The first element of the moving averages set is equal to the average value of the first $k$ elements of the input data set, the second element of the moving averages set is equal to the average value of the second to $(k+1)$ th element of the input data set, etc. If the input data is denoted by $y_{1}, y_{2}, \ldots, y_{n}$, the values of the moving averages are calculated based on the following formula (Eq. 1):

$s_{j+\frac{k-1}{2}}=\frac{1}{k} \sum_{i=j}^{j+k-1} y_{i}, \quad i=1,2, \ldots, n$
$j=1,2, \ldots, n-k+1$ 
The index $j+(k-1) / 2$ will be an integer value if $k$ is an odd number and $s$ is assigned to the time unit $j+(k-1) / 2$. However, if $k$ is an even number this index will not be an integer value, so $s$ is assigned to the time unit $j+k / 2$ and is calculated as follows (Eq. 2):

$S_{j+\frac{k}{2}}=\frac{s_{j+\frac{k-1}{2}}+s_{j+\frac{k+1}{2}}}{2}, j=1,2, \ldots, n-k$

The Moving averages method includes impact of trend. Trend values are calculated by adding the seasonal trend increment to the last calculated moving average. In the case of monthly data, the seasonal trend increment is called the monthly trend increment. The seasonal trend increment is equal to the yearly trend increment divided by the number of seasons per year (there are 12 seasons for 12 -month moving averages). For 6-month moving averages, the half-yearly trend increment is used. Then the seasonal (monthly) trend increment equals the halfyearly trend increment divided by 6 . The yearly (or half-yearly) trend increment is calculated as the difference between the last two average trend increments. The last average trend increment is calculated as the average of the last $k$ calculated moving averages, and the penultimate one as the average value of the penultimate $k$ calculated moving averages.

In addition to trend values, this method includes seasonal variations by computing seasonal indices. The seasonal indices are calculated as average values of seasonal impacts for the same seasons (months) in different years. The seasonal impact is a quotient of input value and moving average for the respective season (month), multiplied by 100 . The forecasted values are obtained by multiplying trend values and seasonal indices for the respective seasons.
This method is very suitable if seasonal variations of input data are obvious and if historical data are available for a longer time. A 12-month moving averages method is the most commonly used in practice. In addition, a 6-month, 3-month, etc. may be used too. The 6-month moving averages method is used in this paper, and the main reason for that is the limited number of monthly data.

\subsection{Exponential Smoothing Methods: Simple Exponential Smoothing Method and Holt-Winters Method}

Exponential Smoothing is generalization of the Moving Averages method. While Moving Averages take into account only the group of recent events, Exponential Smoothing takes into account all values of the time series. It is a forecasting method that weights the observed time series values unequally because more recent observations are weighted more heavily than more remote observations. This unequal weighting is accomplished by one or more smoothing constants, which determine how much weight is given to each observation. It has been found to be most effective when the parameters describing time series may be changing slowly over time.

Exponential Smoothing method represents relatively simple but robust approach to forecasting (Billah et al., 2006). Often, this method can produce approximately good results as some more complex methods. The three most commonly used variations of the Exponential Smoothing method are Simple Exponential Smoothing method (Brown, 1959), Trend-corrected Exponential Smoothing method (Holt, 2004), and HoltWinters method (Winters, 1960).

The Simple Exponential Smoothing method is used in time series with no seasonal effects 
that do not follow any trend, and there are slow changes over time. Forecasting is based on a smoothing equation that depends only on a parameter $\alpha$, which takes a value between 0 and 1 . The value of this parameter should be chosen to minimize the standard error by the Excel solver tool.

The smoothing equation for time instant $T$ takes the following form (Eq. 3):

$$
\begin{aligned}
& l_{t}=\alpha y_{t}+(1-\alpha) y_{t-1}=\alpha y_{t}+(1-\alpha) \alpha y_{t-1}+ \\
& +(1-\alpha)^{2} \alpha y_{t-2}+\cdots+(1-\alpha)^{t} l_{0},
\end{aligned}
$$

where $y_{t}$ is the value of the historical data at time $t$. Parameter $l_{0}$ represents the initial estimate of the mean of the time series and is calculated using the formula (Eq. 4):

$l_{0}=\bar{y}=\frac{\sum_{i=1}^{n} y_{i}}{n}$,

where $y_{i}$ is the historical data values and $n$ is the number of historical data. The forecasted values at time $t(t=1,2,3, \ldots, n)$ are obtained by the following forecast equation (Eq. 5):

$\hat{y}_{t+p}(t)=l_{t}, p=1,2,3 \ldots$

where $p$ represents the future season.

The exponential smoothing is computationally simple and fast, while at the same time this method can perform well in comparison with other complex methods.

The Holt-Winters method works well in practice and particularly is well suited for short-term forecasts (Chatfield and Yar, 1988). Unlike the Simple Exponential Smoothing method, which does not include seasonal influences and does not follow any trend, the Holt-Winters method includes both. There are two variations of this method, Multiplicative Holt-Winters method and Additive Holt-Winters method, which differ in the nature of the seasonal component. The Additive method is preferred when the seasonal variations are roughly constant through the series. Besides that, the series level, growth index and seasonal influence can change slowly over time. The Multiplicative method is preferred when the seasonal variations are changing proportionally to the level of the series. In addition, the time series level, growth index and seasonal influence can change slowly over time. With the Additive method, the seasonal component is expressed in absolute terms in the scale of the observed series, and in the level equation the series is seasonally adjusted by subtracting the seasonal component. Within each year, the seasonal component will add up to approximately zero. With the Multiplicative method, the seasonal component is expressed in relative terms (percentages), and the series is seasonally adjusted by dividing through by the seasonal component. Within each year, the seasonal component will sum up to approximately $L$.

The Holt-Winters seasonal method comprises the forecast equation and three smoothing equations: one for the level $l_{t}$, one for the trend $b$, and one for the seasonal component $s_{t}$, with corresponding smoothing parameters $\alpha, \gamma$ and $\delta$. The main idea of this method is to make forecasts by combining the most recent direct assessment $l_{t}$, with an assessment of the expected increase or decrease, which corresponds to the trend $b_{t}$, all adjusted to the seasonal component $s_{t}$. The trend component of the series is determined using the calculated average of the most recent changes in the level and trend assessment from the previous period. 
The smoothing equation for the seasonal component $s_{t}$ for the additive method is represented by the following formula (Eq. 6):

$s_{t}=\delta\left(y_{t}-l_{t}\right)+(1-\delta) s_{t-L}$,

where $y_{t}$ is historical value at time $t$. We use $L$ to denote the frequency of the seasonality, i.e., the number of seasons in a year. For example, for quarterly data $L=4$, and for monthly data $L=12$.

The smoothing equation for the trend $b_{t}$ is represented by the following formula (Eq. 7):

$b_{t}=\gamma\left(l_{t}-l_{t-1}\right)+(1-\gamma) b_{t-1}$,

The smoothing equation for the level $l_{t}$ is represented by the following formula (Eq. 8):

$l_{t}=\alpha\left(y_{t}-s_{t-L}\right)+(1-\alpha)\left(l_{t-1}+b_{t-1}\right)$,

All three constant smoothing parameters, $a, \gamma$ and $\delta$, takes values between 0 and 1 . The constant $a$ controls the smoothing during the time $t$, where the choice closer to 0 emphasizes the previous values of time series, while value closer to 1 emphasizes the current values, and lessens the importance of previous ones. The constant $\delta$ modifies the seasonal component and the constant $\gamma$ controls data oscillation. The values of parameters $\alpha, \gamma$ and $\delta$ should be chosen to minimize the standard error. Excel solver tool could be used to calculate the optimal values of these parameters.
The forecasted values at time $t(t=1,2,3$, $\ldots, n)$ are obtained by the following forecast equation (Eq. 9):

$\hat{y}_{t+p}(t)=l_{t}+p b_{t}+s_{t+p-L}, \quad p=1,2,3 \ldots$

where $p$ represents the future season.

\subsection{Evaluation of Forecast}

The precision of the forecasted diffusion process is analyzed by performing three tests: MAE (Mean Absolute Error, (Hamilton, 1994)), MAPE (Mean Absolute Percentage Error, (Lewis, 1982)), as well as The Durbin-Watson test (Montgomery, Peck and Vining, 2001). In order to quantify deviation in the data units, MAE and MAPE tests are conducted. MAE and MAPE tests are based on calculating the MAE and MAPE coefficients (Eq. 10-11):

$M A E=\frac{1}{n} \sum_{t=1}^{n}\left|\hat{y}_{t}-y_{t}\right|$

$M A P E=\frac{100}{n} \sum_{t=1}^{n} \frac{\left|y_{t}-\hat{y}_{t}\right|}{\left|y_{t}\right|}$

where $y_{t}$ is historical data value at time $t$, and $\hat{y}_{t}$ is forecasted value at time $t$. If the value of MAE or MAPE coefficients is smaller, then a better fit of the curve is achieved. The values obtained by MAPE test could be also evaluated by using the Lewis scale of a judgment of forecast accuracy (Lewis, 1982), as shown in Table 1:

Table 1

A Scale of Judgment of Forecast Accuracy

\begin{tabular}{|l|l|}
\hline MAPE & Judgment of Forecast Accuracy \\
\hline Less than $10 \%$ & Highly Accurate \\
\hline 11 to $20 \%$ & Good Forecast \\
\hline 21 to $50 \%$ & Reasonable Forecast \\
\hline $51 \%$ or more & Inaccurate Forecast \\
\hline
\end{tabular}

Source: (Lewis, 1982) 
In order to check for the existence of the systematic errors, the third test (DurbinWatson) has been conducted, by using the DW coefficient (Eq. 12):

$D W=2-2 \frac{w}{v}$

where parameters $w$ and $v$ could be obtained by using equations (13) and (14):

$$
\begin{aligned}
& w=\sum_{t=1}^{n-1}\left(y_{t}-\hat{y}_{t}\right)\left(y_{t+1}-\hat{y}_{t+1}\right) \\
& v=\sum_{t=1}^{n}\left(y_{t}-\hat{y}_{t}\right)^{2}
\end{aligned}
$$

where $y_{t}$ is historical data value at time $t$. The values of $D W$ coefficient belong to the range of $0-4$, where the scope $1.5-2.5$ indicates the absence of first-order autocorrelation (the best case is the $D W$ value of 2).

\section{Medium-term Forecasting of the Call Center Calls}

The measurement result of incoming Call Center calls by months during the last two years is shown by Figure 1.

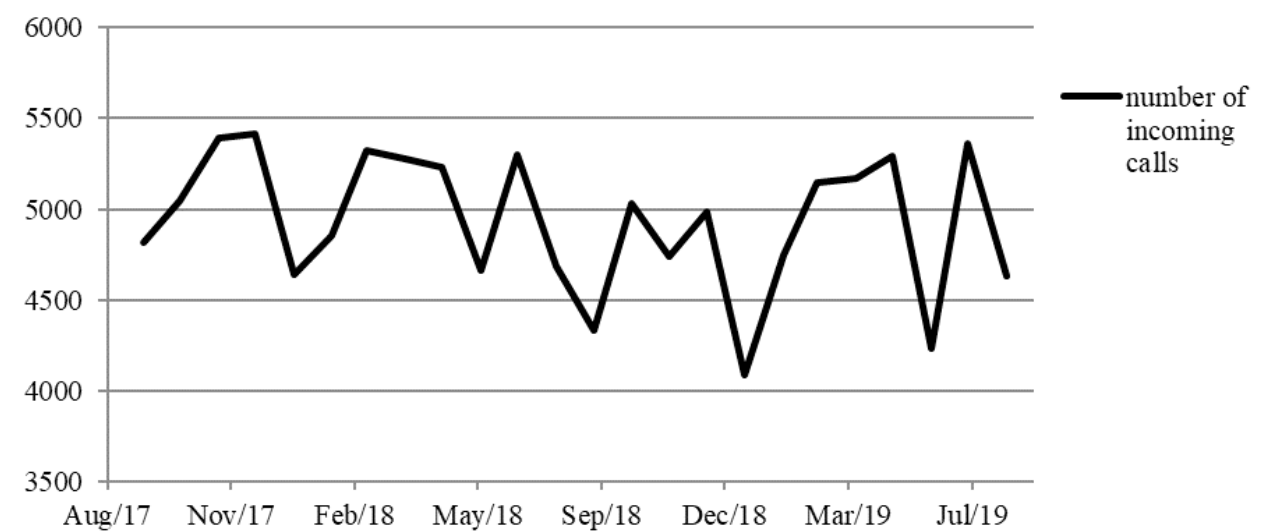

Fig. 1.

The Number of Incoming Call Center Calls

It could be seen that input time series is very dynamic and does not follow any recognizable trend, such as linear or exponential. Also, certain seasonal variations are obvious. For these reasons, it is useful to consider applications by following forecasting methods: the Moving Averages method, Simple Exponential Smoothing method and Additive Holt-Winters method.

The standard procedure for calculating the moving averages has been applied as we explained in Section 3.1. Figure 2 shows historical data of incoming Call Center calls, trend and forecasted number of calls by the 6-month Moving Averages method. It could be notes that the forecasted number of incoming Call Center calls for the previous two years follows the historical data line quite well, that indicates the high accuracy of the forecast. Also, it could be notes that seasonal influences have a much greater impact on the forecast than the trend. 


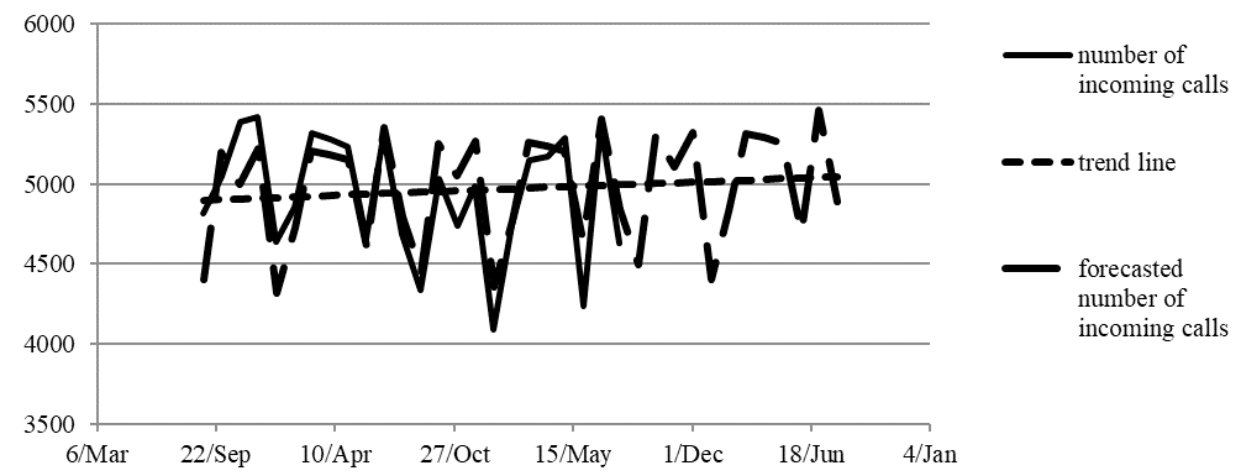

Fig. 2.

Forecasted Number of Calls by the Moving Averages Method

Figure 3 shows historical data of incoming Call Center calls and obtained forecasted number of incoming calls by the Simple Exponential Smoothing method. It could be notes that the forecasted number of incoming Call Center calls going to be constant for the next year, due to the absence of any seasonal influences.

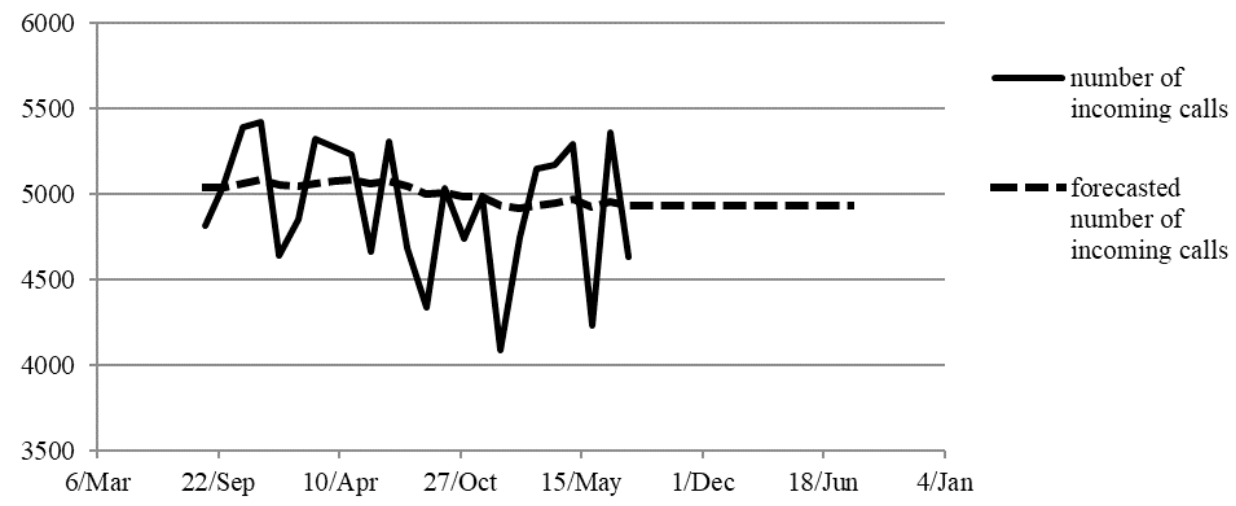

Fig. 3.

Forecasted Number of Calls by the Simple Exponential Smoothing Method

Figure 4 shows historical data of incoming Call Center calls and obtained forecasted number of incoming calls by the Additive Holt-Winters method. 


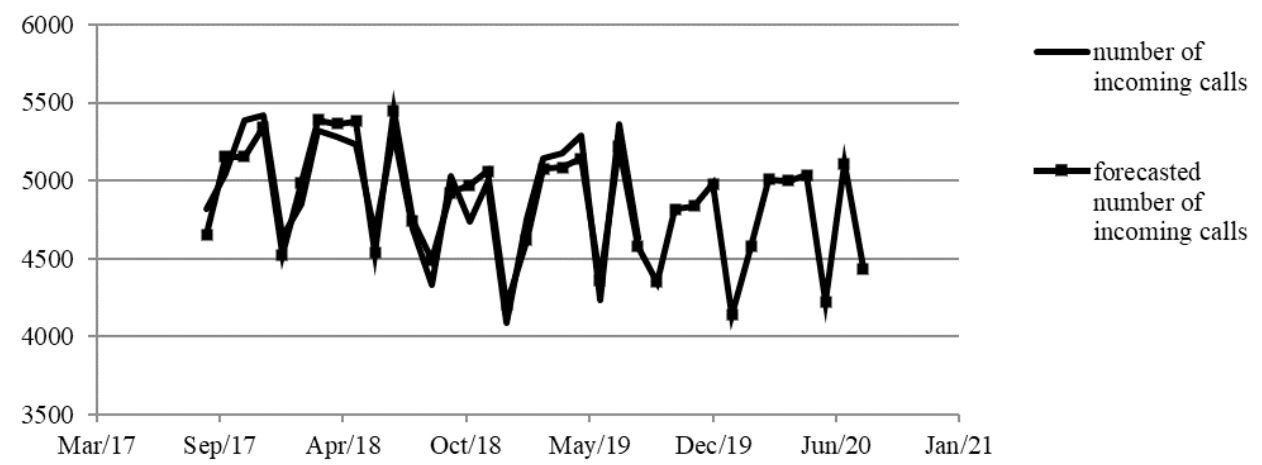

Fig. 4.

Forecasted Number of Calls by the Additive Holt-Winters Method

It can be seen that the forecasted values have excellent matching with historical data in the previous two years.

\section{Forecasting Performance Comparison}

Figure 5 shows the comparison results obtained by the Moving Averages method, Simple Exponential Smoothing method, and Additive Holt-Winters method. This figure shows historical data of Call Center calls in the previous two years, also.

It could be observed that the Additive HoltWinters and Moving Averages method significantly better follow historical data compared to the Simple Exponential Smoothing method. This method could be used only for approximately estimation of the future number of incoming Call Center calls.
Figure 5 shows that the other two methods, the Additive Holt-Winters method and Moving Averages method very good track seasonal variations in the previous two years. Also, it could be notes that the number of calls during the next year forecasted by the Moving Averages method is significantly higher than by the Additive Holt-Winters method. With the Moving Averages method all-time series values have the same impact on the forecast, while with the Additive HoltWinters method the most recent historical data have the greatest impact. In other words, the impact decreases with the distance from the present moment. Looking at historical data, it is noticeable that the number of calls is lower in the second year. For that reason, the Additive Holt-Winters method continues this decreasing trend in the next year. 


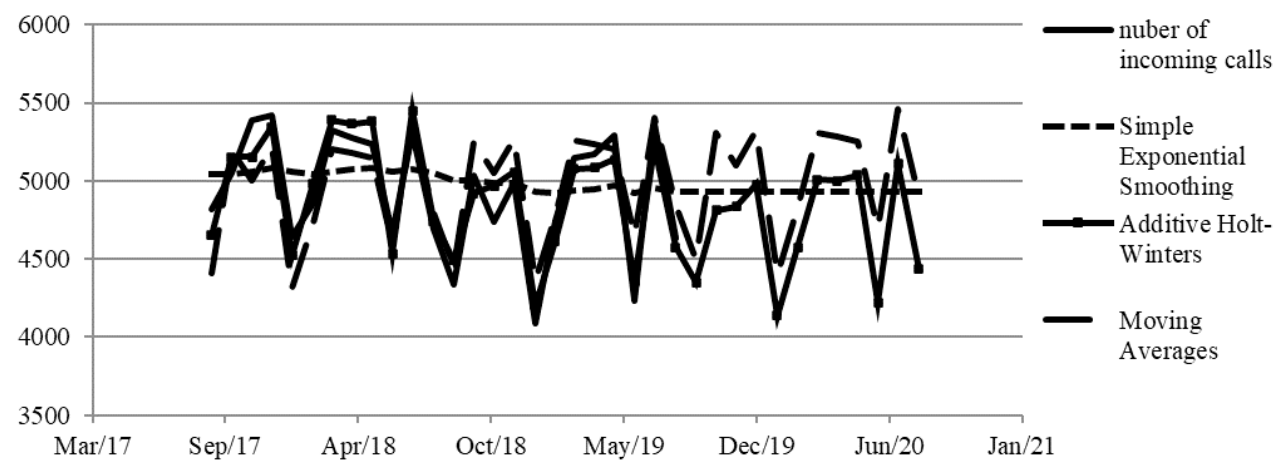

Fig. 5.

Comparison Results

The accuracy of all three forecasting methods can be compared by MAE, MAPE and DW tests. The obtained values of these tests are shown in Table 2.

Table 2

Obtained Values of MAE, MAPE and DW Tests

\begin{tabular}{|c|c|c|c|}
\hline & $\begin{array}{c}\text { Simple Exponential } \\
\text { Smoothing }\end{array}$ & $\begin{array}{c}\text { Additive Holt- } \\
\text { Winters }\end{array}$ & Moving Averages \\
\hline MAE & 300.84 & 121.33 & 178.14 \\
\hline MAPE (\%) & 6.36 & 2.48 & 3.70 \\
\hline DW & 2.21 & 2.28 & 1.31 \\
\hline
\end{tabular}

As can be observed, the lowest mean absolute error is indicated by the Additive HoltWinters method and the largest by the Simple Exponential Smoothing method. In all three cases the values of MAPE test are below $10 \%$, which indicates the high accuracy of the obtained forecasts. However, the Additive Holt-Winters method is extremely suitable and recommended for forecasting highly dynamic time series like incoming Call Center calls. Durbin-Watson test statistic gives unsatisfactory test result for the Moving Averages method.

\section{Conclusion}

The incoming Call Center calls are nonuniform during the year. Occupancy of a Call
Center is significantly higher in some months compared to the other months throughout the year. This fact is directly reflected to optimal resource allocation in a Call Center in order to provide a satisfactory quality of service at all times, without having more agents than necessary. The demand forecast is the main input parameter for capacity resource planning. In this paper, a demand forecast is referred to the short-term forecast of the number of incoming Call Center calls for the next 12 months.

In this paper, we considered three appropriate forecasting methods: the Moving Averages method, Simple Exponential Smoothing method and Additive Holt-Winters method. Based on experimental results this paper 
proposes the Additive Holt-Winters method for forecasting the incoming calls by months. The obtained forecasted values have excellent matching with historical data in the previous two years. This is approved by MAE, MAPE and DW tests. Since the selected forecasting method has shown very good performance in such a dynamic time series, we would like to recommend this forecasting method for the other applications with dynamic time series such as IP traffic.

In the future, our work could be extended to include some other forecasting methods suitable for dynamic time series, such as Trend-corrected Exponential Smoothing method, Multiplicative Holt-Winters method, neural networks, Box-Jenkins, etc. Also, it is possible to apply some additional tests for the accuracy of the forecast.

\section{Acknowledgements}

This paper has been partially supported by the Ministry of Education, Science and Technological Development of the Republic of Serbia project under No. 32025.

\section{References}

Billah, B.; King, M.; Snyder, R.; Koehler, A. 2006. Exponential smoothing model selection for forecasting, International Journal of Forecasting 22(2): 239-247.

Brockwell, D. 2002. Introduction to Time Series and Forecasting. Springer-Verlag, New York. 425 p.

Brown, R. G. 1959. Statistical forecasting for inventory control. McGraw Hill, New York. 233 p.

Chatfield, C.; Yar, M. 1988. Holt-Winters Forecasting: Some Practical Issues, Journal of the Royal Statistical Society 32(2): 101-239.
Gans, N.; Koole, G.; Mandelbaum, A. 2003. Telephone call centers: tutorial, review and research prospects, Manufacturing and Service Operations Management 5(2): 79-141.

Hamilton, J. D. 1994. Time Series Analysis. Princeton University Press 11(3): 625-630.

Holt, C. C. 2004. Forecasting Trends and Seasonal by Exponentially Weighted Averages, International Journal of Forecasting 20(1): 5-10.

Kovačić, Z. 1998. Time Series Analysis [In Serbian: Analiza Vremenskih serija]. Ekonomski fakultet, Beograd. 354 p.

Lepojević, V.; Anđelković-Pešić, M. 2011. Forecasting electricity consumption by using Holt-Winters and Seasonal Regression models, Facta Universitatis Series: Economics and Organization 8(4): 421 - 431.

Lewis, C. D. 1982. International and Business Forecasting Methods. Butterworths, London. 305 p.

Montgomery, D. C.; Peck, E. A.; Vining, G. G. 2001. Introduction to Linear Regression Analysis. Wiley, New York. $688 \mathrm{p}$.

Radojičić, V.; Bakmaz, B. 2010. Application of quantitative forecasting methods in telecommunications [In Serbian: Primena kvantitativnih metoda prognoziranja u telekomunikacijama]. Saobraćajni fakultet, Beograd. 94 p.

Radojičić, V.; Marković, G.; Radonjić V. 2010. A new method for estimating the real number of callers for call centre staffing optimisation, International Journal Engineering Management and Economics (IJEME) 1(4): 321-341.

Winters, P. R. 1960. Forecasting sales by exponentially weighted moving averages, Management Science 6(3): 324-342. 